\title{
Molecular Interactions of Carbapenem Antibiotics with the Multidrug Efflux Transporter AcrB of Escherichia coli
}

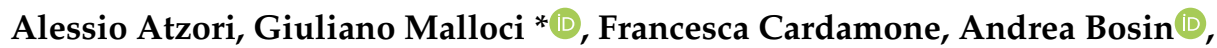 \\ Attilio Vittorio Vargiu *(D) and Paolo Ruggerone \\ Department of Physics, University of Cagliari, 09042 Monserrato (CA), Italy; alessio.atzori@dsf.unica.it (A.A.); \\ francesca.cardamone@dsf.unica.it (F.C.); andrea.bosin@dsf.unica.it (A.B.); paolo.ruggerone@dsf.unica.it (P.R.) \\ * Correspondence: giuliano.malloci@dsf.unica.it (G.M.); vargiu@dsf.unica.it (A.V.V.); \\ Tel.: +39-070-675-4911 (G.M. \& A.V.V.)
}

Received: 14 January 2020; Accepted: 26 January 2020; Published: 29 January 2020

\begin{abstract}
The drug/proton antiporter AcrB, engine of the major efflux pump AcrAB(Z)-TolC of Escherichia coli and other bacteria, is characterized by its impressive ability to transport chemically diverse compounds, conferring a multi-drug resistance (MDR) phenotype. Although hundreds of small molecules are known to be AcrB substrates, only a few co-crystal structures are available to date. Computational methods have been therefore intensively employed to provide structural and dynamical fingerprints related to transport and inhibition of AcrB. In this work, we performed a systematic computational investigation to study the interaction between representative carbapenem antibiotics and AcrB. We focused on the interaction of carbapenems with the so-called distal pocket, a region known for its importance in binding inhibitors and substrates of AcrB. Our findings reveal how the different physico-chemical nature of these antibiotics is reflected on their binding preference for AcrB. The molecular-level information provided here could help design new antibiotics less susceptible to the efflux mechanism.
\end{abstract}

Keywords: antibiotic resistance; Gram-negative bacteria; resistance nodulation-cell division transporters; AcrB; molecular docking; molecular dynamics simulations; binding free energy calculations

\section{Introduction}

The increased number of MDR Gram-negative bacterial strains, along with the reduced efficacy of available antibiotics, constitute a significant concern for public health [1-3]. A prominent role in the MDR phenotype is played by resistance nodulation-cell division (RND) efflux pumps [4-12], complex poly-specific transporters involved in the extrusion of a wide range of chemically unrelated compounds. The inner membrane drug/proton antiporter $\mathrm{AcrB}$, part of the major $\mathrm{Acr} A B(\mathrm{Z})$-TolC efflux system of Escherichia coli, is one of the most investigated members of the RND superfamily [6,13-19]. Structurally, AcrB is a homotrimer and each of its protomers is composed of a transmembrane (TM) domain (where a proton gradient provides the energy needed for substrate translocation [20]), a porter domain (responsible for the recognition, uptake, and first transport of substrates), and a funnel domain (constituted by a channel linked to partner proteins AcrA and TolC) (Figure 1). 


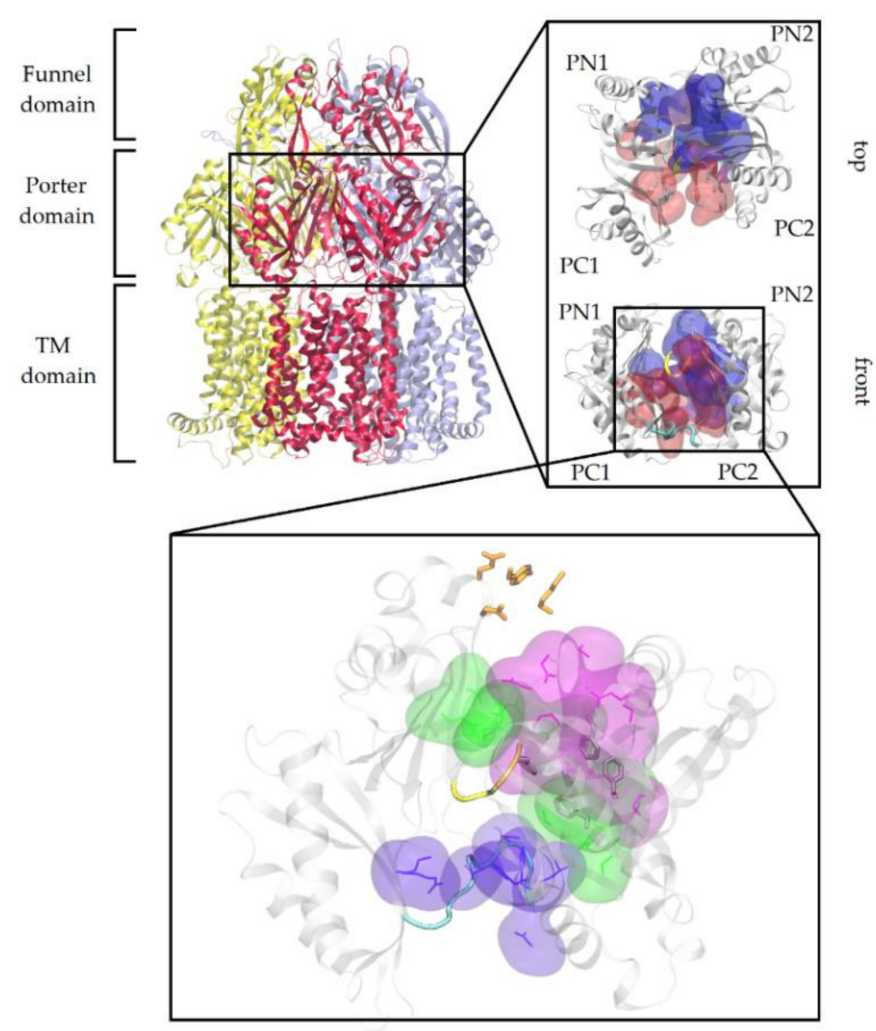

Figure 1. View of the AcrB trimer. The access, binding, and extrusion protomers of AcrB are represented as yellow, red, and ice blue ribbons, respectively. The inset shows a front and top magnification of the porter domain of the T monomer of AcrB highlighting PN1, PN2, PC1, and PC2 subdomains and the access and deep binding pockets represented as red and blue surfaces, respectively. The second inset shows the main regions of interest discussed in this study, as reported in Table S1. The switch-loop and the bottom loop are displayed as yellow and cyan tubes, respectively. HP-trap and exit gate residues are shown as gray and orange sticks, respectively. Interface, groove, and cave residues are represented as violet, green, and purple surfaces and lines respectively. Protein residues lining each region are reported in Table S1.

Both a symmetric and an asymmetric conformation of AcrB, thought to represent its resting and active states respectively, have been identified by X-ray crystallography $[13,14,16,21-24]$. In the asymmetric structure, each protomer is found in a different conformational state defined as loose (L), tight $(\mathrm{T})$, and open $(\mathrm{O})[13,18,19]$. This finding suggested that the efflux of substrates is induced by a functional rotation mechanism [13-15], where each protomer can alternatively assume the above conformations in concert with the others. This hypothesis has been supported by subsequent experimental $[25,26]$ and computational studies [27-31]. Additionally, the available asymmetric structures of AcrB allowed for the identification of specific substrate recognition sites: (i) the access pocket (AP), located in the vestibule region between PC1 and PC2 subdomains and open in protomers L and T [16,32]; (ii) the distal pocket [16,32], located more closely to the funnel domain and open only in the $\mathrm{T}$ protomer (hereafter $\mathrm{DP}_{\mathrm{T}}$; see Table $\mathrm{S} 1$ for the list of residues belonging to different regions of AcrB). Recently, a new binding site localized in the transmembrane region has been identified [26].

Due to its crucial position in the entire efflux pathway, the $\mathrm{DP}_{\mathrm{T}}$ is supposed to interact with all $\mathrm{AcrB}$ substrates along their transport pathway, independently of their molecular size and physico-chemical features. This hypothesis has been recently supported by the publication of the co-crystal structures of high molecular mass compounds bound within the $\mathrm{DP}_{\mathrm{T}}$ of $\mathrm{MexB}$, the homologous protein of $\mathrm{Acr} B$ in Pseudomonas aeruginosa [33]. The portion of the $\mathrm{DP}_{\mathrm{T}}$ comprising multiple phenylalanine residues $(136,628,610,615,628)$ and named "hydrophobic trap" (HP-trap) [34], is a crucial recognition site 
for AcrB inhibitors [35,36], and was also shown to interact with several substrates [16,32,37-39]. The interface between access and distal pockets is constituted by a glycine rich loop (aka "switch loop"), which regulates the transition of substrates towards the $\mathrm{DP}_{\mathrm{T}}[16,32,40]$. A "bottom-loop", which could play a role as well in the recognition and transport of substrates, is found oppositely to the switch-loop within the access pocket [40-42] (Figure 1).

A detailed understanding of the relationship between physico-chemical properties of antibiotics and their propensity to be expelled by efflux pumps is relevant to understand the mechanisms of polyspecific transport and can be very informative for drug design campaigns. Due to the complexity of RND transporters and the difficulty of producing co-crystals, only a few X-ray structures of the asymmetric trimer of AcrB bound to substrates have been reported to date [13-16,18,20,26,32,34]. The characteristics of compounds co-crystallized so far led to the conclusion that a certain degree of hydrophobicity is required for substrates of AcrB [43]. Computational studies can provide atomistic information on the dynamics of biomolecular complexes. As such, they represent a viable alternative to gain insights into the binding of compounds to AcrB. One of the earlier studies on this protein identified two distinct sub portions of the $\mathrm{DP}_{\mathrm{T}}$, called "groove" and "cave" $[44,45]$. The groove region is located in the upper portion of the $\mathrm{DP}_{\mathrm{T}}$, close to the so-called exit gate (EG, Table S1) [15], while the broader cave region is located at the bottom of the $\mathrm{DP}_{\mathrm{T}}$. Later, molecular dynamics (MD) simulations have been used to investigate substrate binding and specificity of AcrB $[37,38,46]$, as well as to unveil the link between structural-chemical fingerprints of compounds and physico-chemical properties of the multidrug binding sites of AcrB [42,47]. These studies revealed that compounds can preferentially bind to the cave, to the groove or to both regions, but no clear rules were identified explaining the binding preferences of different compounds. Indeed, almost iso-energetic binding modes were predicted for the same ligand, a result compatible with the so-called oscillation hypothesis [19], according to which AcrB substrates oscillate between different binding modes with similar affinity within the $\mathrm{DP}_{\mathrm{T}}$.

Among the different families of in-use antibiotics, carbapenems represent a primary resource for the treatment of severe bacterial infections $[48,49]$. Unfortunately, several carbapenem-resistant Gram-negative strains emerged over the years, for which there is evidence that these antibiotics can be recognized and extruded by RND efflux pumps [50-52]. These conclusions usually relied on the comparison between minimum inhibitory concentration (MIC) measurements obtained for wild-type and efflux-pumps deficient strains. However, MIC values are strongly influenced by the interaction of the drug with many other bacterial components: for instance, the membrane permeability, heavily dependent on intrinsic drug properties, can definitely surpass the specific contribution of active efflux to MDR [53,54]. As an example, while meropenem is a good substrate of the MexAB-OprM efflux pump from P. aeruginosa and imipenem resistance to this organism appears to be predominantly caused by changes in outer membrane pores, the impact of AcrAB-TolC on the activity of IMI and MER in E. coli is still under debate [55]. In this context, direct efflux and affinity measurements [56-58], combined with a molecular picture of the interaction between these compounds and AcrB, would be pivotal for the design of more effective antibiotics starting from validated and widely used scaffolds.

Along these lines, we report here a comparative computational investigation of the interactions between selected carbapenem antibiotics, such as biapenem (BIA), doripenem (DOR), ertapenem (ERT), faropenem (FAR), imipenem (IMI), meropenem (MER), panipenem (PAN), and tomopenem (TOM) (Figure 2), and the $\mathrm{DP}_{\mathrm{T}}$ of AcrB. Half of the selected carbapenems (namely DOR, ERT, IMI, and MER) are currently in clinical use. As a reference, two efflux pump inhibitors (MBX3132 and D13-9001, hereafter MBX and P9D respectively) and two known substrates of AcrB (rhodamine-6G and minocycline, hereafter RDM and MIN) was investigated with the same computational protocol adopted for carbapenems (Figure 2). 


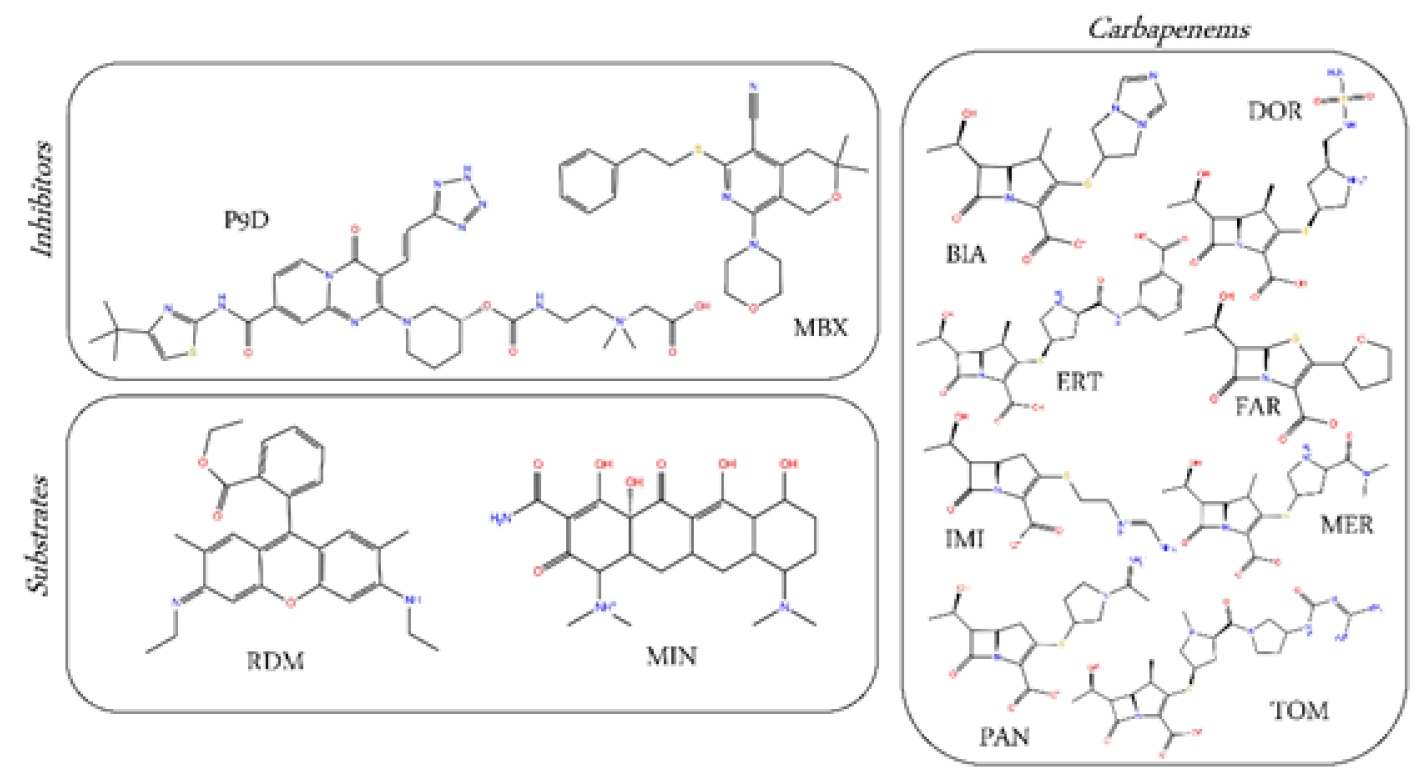

Figure 2. Two-dimensional (2-D) structures of the substrates/inhibitors co-crystallized with AcrB and of the selected carbapenems antibiotics considered in this study.

The outcomes of this work led to the identification of binding modes and specific interactions between chemically related molecules and different sub-pockets within the $\mathrm{DP}_{\mathrm{T}}$ of AcrB. This study contributes to explain the detailed interactions established by different carbapenem antibiotics with the $\mathrm{DP}_{\mathrm{T}}$ of $\mathrm{AcrB}$ in terms of their intrinsic physico-chemical properties. A retrospective analysis of the previous literature on other antimicrobial compounds [44] revealed the general applicability of our findings $[35,55,59-61]$.

\section{Results}

In continuation of our previous studies $[37,55,59,60]$, we combined molecular docking, MD simulations, binding free-energy and hydrophobic/hydrophilic surface-matching calculations, and solvation analyses. Namely, for each compound, one single mode of binding within the $\mathrm{DP}_{\mathrm{T}}$ of $\mathrm{AcrB}$ was found from blind docking calculations. These docking poses (and the crystallographic configurations considered) were used as starting conformations for 100 ns-long all-atom MD simulations in water solution followed by a cluster analysis of the trajectory. Binding free-energy calculations were thus performed on the conformations extracted from the most populated conformational cluster. From the cluster representative configuration, the hydrophobic $\left(\mathrm{SM}_{\mathrm{L}}\right)$ and hydrophilic $\left(\mathrm{SM}_{\mathrm{H}}\right)$ matching coefficients between the interaction surfaces of the compound and the $\mathrm{DP}_{\mathrm{T}}$ of $\mathrm{AcrB}$ were computed: both quantities range from (no matching) to 1 (full matching). Additionally, the de-solvation of each compound upon binding to AcrB was also estimated from MD simulations (see Materials and Methods for details).

In the following paragraphs, we first present the results for substrates and inhibitors co-crystallized with AcrB; next, we discuss the data obtained for the carbapenem antibiotics considered in this work. The starting X-ray crystal configurations and the selected docking poses as well as the structural cluster representative of each MD simulation can be visualized online at the web address www.dsf.unica.it/translocation/docking. To rationalize our results in terms of specific physico-chemical features of the compounds, we report some molecular properties in Table S2. Molecular weights (MWs) are approximately in the range $300-500 \mathrm{Da}$, while the total charge ranges from -1 to +1 including several neutral zwitterionic species. Based on available MICs data (compiled in Table S3), the AcrAB-TolC efflux pump would recognize and expel the considered compounds. However, as specified in the 
Introduction, a detailed atomistic description of the interactions of different compounds within the extrusion channel of AcrB cannot be achieved with current experimental techniques.

\subsection{Substrates and Inhibitors Co-Crystallized with AcrB}

Comparative data available for the crystallographic poses of substrates and inhibitors bound to the $\mathrm{DP}_{\mathrm{T}}$ of AcrB are summarized in Table 1. Two substrates MIN (PDB ID: 4DX5 [32]) and RDM (PDB ID: 5ENS [36]), and the two inhibitors P9D (PDB ID: 3W9H [34]) and MBX (PDB ID: 5ENQ [36]) were considered. MD simulations revealed overall stability of the crystallographic poses (Figure S1), as further confirmed by the cluster analysis of the trajectory which predicted one single conformational cluster in all cases. The root-mean-square displacement (RMSD) of the cluster representative from the starting X-ray configuration has the value of 2.1, 4.0, 1.7, and 2.4 for MIN, RDM, MBX, and P9D, respectively (Figure S2). The hydrophobic surface-matching analysis showed that the lipophilic surface matching $\left(\mathrm{SM}_{\mathrm{L}}\right)$ plays an important role in the interaction observed for all the compounds (values between 0.80 and 0.96). On the contrary, the hydrophilic surface matching $\left(\mathrm{SM}_{\mathrm{H}}\right)$ is found to vary from 0.01 (RDM) to 0.91 (P9D) according to the very different chemical nature of these compounds as quantified by the octanol/water partition coefficient $\log \mathrm{P}$ (Table S2).

Table 1. List of substrates and inhibitors co-crystallized with AcrB and considered in this study, along with their molecular identifier and molecular weight (MW). The central columns report the hydrophobic $\left(\mathrm{SM}_{\mathrm{L}}\right)$, and hydrophilic surface matching $\left(\mathrm{SM}_{\mathrm{H}}\right)$, and the percentage of water molecules in the first solvation shell (Hyd) calculated against molecular dynamics (MD) simulations of the corresponding compound in water solution. The remaining columns report respectively the free energy of binding $\left(\Delta G_{b}\right)$ for the most populated cluster trajectory identified for each simulation, and the percentage contribution to $\Delta \mathrm{G}_{\mathrm{b}}$ from residues lining the interface between access pocket and $\mathrm{DP}_{\mathrm{T}}$, the cave, and the groove regions of the $\mathrm{DP}_{\mathrm{T}}$ (see Figure 1 and residues list in Table S1).

\begin{tabular}{|c|c|c|c|c|c|c|c|c|c|}
\hline \multirow{2}{*}{$\begin{array}{l}\text { Co-Crystallized } \\
\text { AcrB Compound }\end{array}$} & \multirow{2}{*}{ ID } & \multirow{2}{*}{$\begin{array}{l}\text { MW } \\
\text { (Da) }\end{array}$} & \multirow{2}{*}{$\mathbf{S M}_{\mathrm{L}}$} & \multirow{2}{*}{$\mathbf{S M}_{\mathbf{H}}$} & \multirow{2}{*}{$\begin{array}{c}\text { Hyd } \\
(\%)\end{array}$} & \multirow{2}{*}{$\underset{\text { (kcal/mol) }}{\Delta \mathrm{G}_{\mathrm{b}}}$} & \multicolumn{3}{|c|}{ Contribution to $\Delta \mathrm{G}_{\mathrm{b}}(\%)$} \\
\hline & & & & & & & Interface & Cave & Groove \\
\hline Rhodamine-6G & RDM & 444 & 0.80 & 0.01 & $35 \pm 8$ & $-38.3 \pm 3.3$ & 2 & 5 & 29 \\
\hline Minocycline & MIN & 458 & 0.80 & 0.70 & $34 \pm 8$ & $-29.3 \pm 4.8$ & 1 & 5 & 53 \\
\hline MBX3132 & MBX & 495 & 0.87 & 0.63 & $30 \pm 8$ & $-53.6 \pm 4.6$ & 2 & 18 & 24 \\
\hline D13-9001 & P9D & 693 & 0.96 & 0.91 & $40 \pm 7$ & $-52.3 \pm 4.9$ & 2 & 22 & 25 \\
\hline
\end{tabular}

No clear trend was found between the dehydration of compounds and their classification as substrates or inhibitors of $\mathrm{AcrB}$ (Table 1). Given the highly hydrophobic nature of the $\mathrm{DP}_{\mathrm{T}}$, a consistent fraction of waters was lost in all cases (from $60 \%$ to $70 \%$ compared to the value in bulk water).

As to the strength of the interaction, the inhibitors show higher affinities (larger negative values of the binding free energy $\Delta \mathrm{G}_{\mathrm{b}}$ ) in comparison to substrates. In order to investigate more deeply the reason for this difference, we analysed the per-residue contributions to $\Delta G_{b}$ focusing on residues lining three well-defined portions of the pocket so as to locate spatially the binding mode of each compound: the entrance of the $\mathrm{DP}_{\mathrm{T}}$ (or the Interface between access pocket and $\mathrm{DP}_{\mathrm{T}}$ ), and the cave and groove regions defined in [44] (Table S1). The contribution to $\Delta \mathrm{G}_{\mathrm{b}}$ of the different regions of the $\mathrm{DP}_{\mathrm{T}}$ reported in Table 1 reveal that: a) the interface turns out to contribute almost nothing $(1-2 \%)$ to the stabilization of both substrates and inhibitors; b) MIN and RDM appear to be prevalently groove binders (contribution in the range 30-50\%); c) MBX and P9D bind the entire pocket, interacting with both the cave and the groove regions (energetic contribution of about $20 \%$ for both cave and groove). The detailed pattern observed (Figure 3) reveals the prominent role played by PHE178, ILE277, VAL612, and PHE615 (groove) in stabilizing the binding of all compounds, as well as the contribution of the hydrophobic residues PHE136, VAL139, TYR327, VAL571, and PHE628 (cave) only to the stabilization of inhibitors. Importantly, not only hydrophobic residues (especially the phenylalanines of the HP-trap), but also polar (ARG620) and weakly polar (SER287) residues are found to be relevant. All of the above findings are consistent with previous investigations [35,37] and available structural and biophysical 
data, validating the adopted computational protocol for a systematic investigation of the binding of carbapenems to the $\mathrm{DP}_{\mathrm{T}}$ of AcrB.

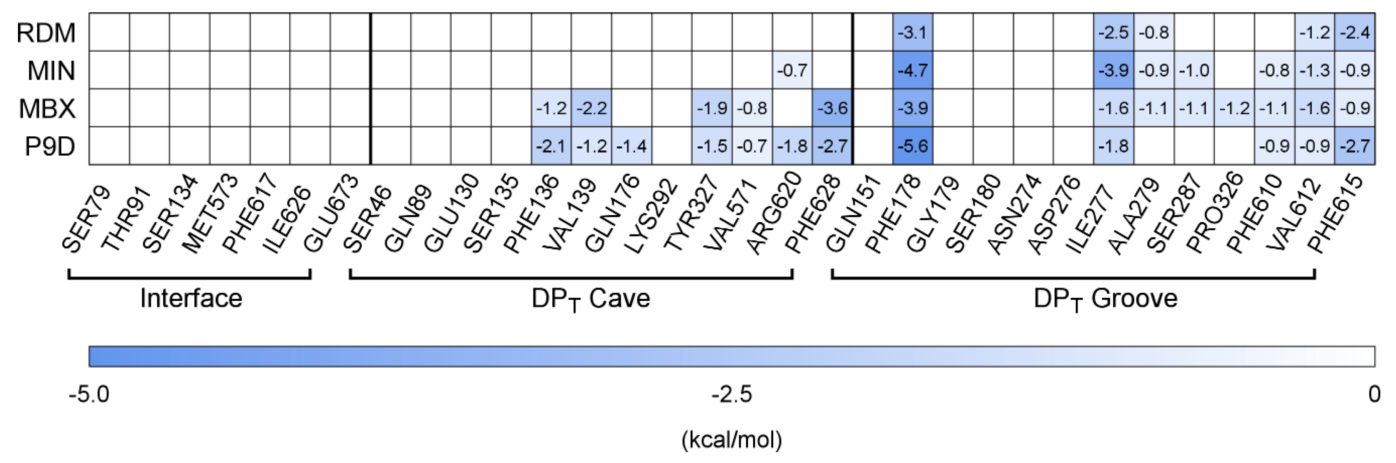

Figure 3. Per-residue contributions to the free-energy of binding (kcal/mol) evaluated for the considered substrates and inhibitors co-crystallized with AcrB. Only residues contributing more than $\mathrm{kT}(\sim 0.6 \mathrm{kcal} / \mathrm{mol}$ at $310 \mathrm{~K})$ are reported. Residues of the interface, $\mathrm{DP}_{\mathrm{T}}$ cave and $\mathrm{DP}_{\mathrm{T}}$ groove are colored with different tones of blue according to their individual contribution to the overall $\Delta \mathrm{G}_{\mathrm{b}}$.

\subsection{Carbapenems}

Given the absence of experimental data concerning the molecular interaction of carbapenems with the $\mathrm{DP}_{\mathrm{T}}$ of $\mathrm{AcrB}$, the same computational investigation described for known inhibitors and substrates of this transporter was performed for selected antibiotics of this family.

Table 2 summarizes the results obtained for the carbapenems, sorted by increasing MW. MD simulations revealed that few compounds remained close to the starting configuration (MER, PAN, DOR, see Figure S3), while consistent mobility was observed for the remaining compounds, with RMSDs ranging from 4-5 $\AA$ (FAR, BIA, ERT) up to 5-6 $\AA$ (IMI, TOM). Contrary to what has been observed for known substrates and inhibitors of $A c r B$, in the case of carbapenems the hydrophilic surface matching seems to be more relevant than the lipophilic matching (on average, we found higher values of $\mathrm{SM}_{\mathrm{H}}$ as compared to $\mathrm{SM}_{\mathrm{L}}$, and in 3 out of 8 cases the latter value almost vanishes). Measured dehydration for carbapenems is similar to that observed for substrates and inhibitors only for FAR, IMI and MER (60-70\% of water molecules lost). The other members of the class show less pronounced dehydration in the range $50-60 \%$. The values of $\Delta \mathrm{G}_{\mathrm{b}}$ computed for carbapenems are of the same order of magnitude as that of MIN (about $-30 \mathrm{kcal} / \mathrm{mol}$ ). Notably, in agreement with the results of our previous study [55], MER displays a moderately higher affinity than IMI for the $\mathrm{DP}_{\mathrm{T}}$ of $\mathrm{AcrB}$ ( $-31 \mathrm{vs} .-25 \mathrm{kcal} / \mathrm{mol})$, coupled with reduced mobility. Interestingly, some qualitative trends appear when considering the different contributions to the total $\Delta \mathrm{G}_{\mathrm{b}}$ of the interface, the cave, and the groove regions of the $\mathrm{DP}_{\mathrm{T}}$. More specifically, the contribution of the interface, which was almost null for known substrates and inhibitors, remains relatively small but roughly anti-correlated to the MW, ranging from $\sim 10 \%$ for the smallest compound to zero for the largest one. Except for DOR, MER, and TOM, the contribution of the cave falls in the range $20-30 \%$, on average exceeding the values registered for inhibitors $(\sim 20 \%)$. On the contrary, while for smaller carbapenems the contribution of groove residues is reduced $(\sim 5 \%)$, for bulkier compounds we observe the same order of magnitude found for substrates and inhibitors $(\sim 30 \%)$. The only exception to the above trend is represented by TOM, whose cave and groove contributions are similar and relatively small $(\sim 15 \%)$. 
Table 2. Structural and energetic features of the carbapenem-AcrB interaction. See Table 1 for details.

\begin{tabular}{cccccccccc}
\hline $\begin{array}{c}\text { Carbapenem } \\
\text { Antibiotic }\end{array}$ & ID & $\begin{array}{c}\mathbf{M W} \\
\mathbf{( D a})\end{array}$ & $\mathbf{S M}_{\mathbf{L}}$ & $\mathbf{S M}_{\mathbf{H}}$ & $\begin{array}{c}\mathbf{H y d} \\
\mathbf{( \% )}\end{array}$ & $\begin{array}{c}\Delta \mathbf{G}_{\mathbf{b}} \\
\mathbf{( k c a l} / \mathbf{m o l})\end{array}$ & \multicolumn{2}{c}{$\begin{array}{c}\text { Contribution to } \Delta \mathbf{G}_{\mathbf{b}}(\%) \\
\text { Interface }\end{array}$} & $\begin{array}{c}\text { Cave } \\
\mathbf{G r o o v e}\end{array}$ \\
\hline Faropenem & FAR & 284 & 0.45 & 0.81 & $27 \pm 9$ & $-25.5 \pm 3.5$ & 12 & 31 & 5 \\
\hline Imipenem & IMI & 299 & 0.11 & 0.21 & $37 \pm 13$ & $-25.1 \pm 4.7$ & 6 & 24 & 4 \\
\hline Panipenem & PAN & 339 & 0.01 & 0.80 & $50 \pm 11$ & $-27.6 \pm 5.0$ & 4 & 23 & 5 \\
\hline Biapenem & BIA & 350 & 0.04 & 0.60 & $45 \pm 13$ & $-30.5 \pm 5.5$ & 5 & 20 & 7 \\
\hline Meropenem & MER & 383 & 0.29 & 0.73 & $35 \pm 8$ & $-30.8 \pm 4.3$ & 1 & 15 & 29 \\
\hline Doripenem & DOR & 420 & 0.35 & 0.84 & $47 \pm 10$ & $-33.6 \pm 5.5$ & 0 & 14 & 33 \\
\hline Ertapenem & ERT & 475 & 0.30 & 0.51 & $42 \pm 9$ & $-33.8 \pm 7.8$ & 3 & 28 & 31 \\
\hline Tomopenem & TOM & 539 & 0.01 & 0.54 & $50 \pm 9$ & $-32.6 \pm 6.7$ & 0 & 15 & 12 \\
\hline
\end{tabular}

The above analysis is complemented by the detailed per-residue contributions reported inFigure 4 , which reveal a thermodynamic pattern overall different from that shown in Figure 3 for known substrates and inhibitors of AcrB. Firstly, for a few low-MW carbapenems a non-negligible contribution to $\Delta \mathrm{G}_{\mathrm{b}}$ comes from some residues of the Interface region (PHE617 belonging to the switch-loop between the AP and the DP, or THR91, MET573, and ILE626). Secondly, stabilization of low-MW carbapenems appears to be due mostly to residues from the cave, including several hydrophobic but also polar and charged sidechains. Thirdly, at increasing MW, the compounds appear to be more and more buried within the whole $\mathrm{DP}_{\mathrm{T}}$, stabilized by residues from both cave and groove residues (similar to what is observed for some inhibitors [34,36]; see also Figure S4). Overall, PHE617 of the Interface, PHE136 and ARG620 of the cave, and PHE178, ILE277, and PHE615 of the groove contribute mostly to the free energy of binding of the carbapenems considered in this work.

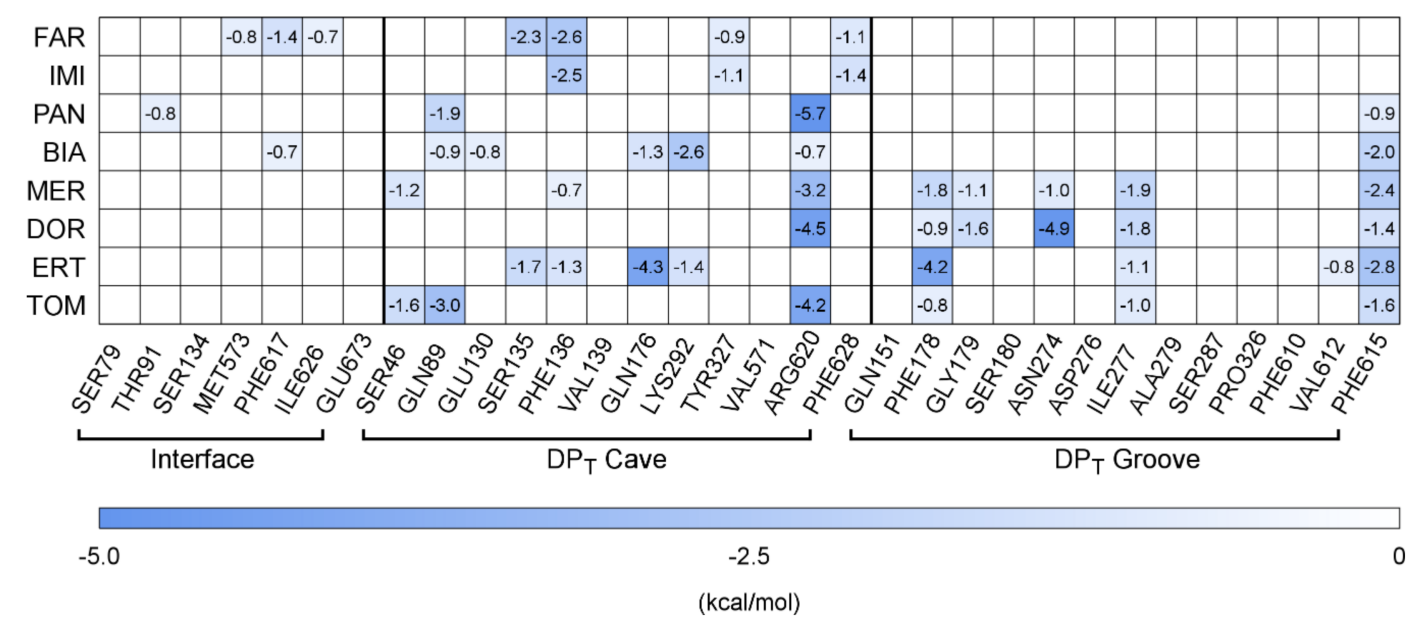

Figure 4. Per-residue contributions to the free-energy of binding (kcal/mol) of carbapenems to AcrB. See Figure 3 for further details.

As expected, consistently with the mainly lipophilic nature of the $\mathrm{DP}_{\mathrm{T}}$, hydrophobic residues are found to be the majority. Additional residues contributing to the stabilization of some carbapenems, such as GLN89, SER135, TYR327, and ASN274, are known to play a key role in the efflux of substrates mediated by AcrB [62].

\section{Discussion}

Despite the recognized role of RND efflux systems in multi-drug resistance to antibiotics, a quantitative assessment of their contribution remains challenging due to the difficulties in 
the determination of the efflux kinetics of most substrates of AcrB and homologous RND transporters $[58,63,64]$. While for other drugs the binding to AcrB has been characterized structurally $[32,34,36]$ and/or by means of site-directed mutagenesis [65-67] to the best of our knowledge for the carbapenems investigated in this study, the available data possibly related to efflux-mediated resistance are the variations in the MIC values of antibiotics upon deletion of the outer membrane transporter TolC and/or AcrB (or homologous proteins see e.g., [68-70]). These data, however, notoriously reflect additional processes involving antibiotics and other bacterial components [53].

Furthermore, the challenges of obtaining experimental structures of RND transporters in complex with their substrates must be taken into account in this scenario [8]. Henceforth, several computational labs made efforts at determining the molecular interactions between these multi-drug proteins and different compounds, including antibiotics and inhibitors [37,42,44,47,71,72] (for a recent review, see [73] and references therein). The work presented here can be framed in this context and was motivated by the purpose of providing information about the possible interaction between selected carbapenems and AcrB of E. coli. Indeed, to the best of our knowledge, no direct efflux measurements are available for this class of compounds. In contrast, whereas kinetic efflux data are available for other families of $\beta$-lactam antibiotics $[58,63]$.

The binding propensity of carbapenems to the $\mathrm{DP}_{\mathrm{T}}$ of $\mathrm{AcrB}$ as well as their interaction patterns revealed several remarkable features. First, along with the MD simulations, all compounds but imipenem adopted a relatively stable (along the timescale of tens of ns) binding position within the $\mathrm{DP}_{\mathrm{T}}$ of AcrB, regardless of the displacement from the initial docking pose (Figures S3 and S4). A comparable dynamic behavior for imipenem (supposedly not an AcrB substrate) was observed in our previous study [55]. Second, we found an interesting correlation between the MW of the compounds and their binding preference to previously identified sub-pockets within the DP. Namely, the groove seems to contribute to the binding of medium/high MW substrates, while smaller compounds tend to cluster within the cave and interact marginally with the AP/DP Interface. The only exception to this rule seems to be ertapenem, for which the cave and the groove contribute to a similar extent to the binding, mostly because of a significant role of PHE178 for the stabilization of this compound. Tomopenem turns out to be the compound for which the residues from groove, cave, and interface contribute less to the binding. Third, another peculiar property for the carbapenems family highlighted by the MD simulations, appears to be the ability of the drugs in the hydrophobicity screen of the $\mathrm{DP}_{\mathrm{T}}$. The higher $\mathrm{SM}_{\mathrm{H}}$ values found for the carbapenems in comparison to $\mathrm{SM}_{\mathrm{L}}$ values (Table 2) indicate a preference for a hydrophilic complementary interactions surface between the drug and the protein, although the prevalent composition of the $\mathrm{DP}_{\mathrm{T}}$ is hydrophobic.

In a broader perspective, our findings are in agreement with previous results by Takatsuka and Nikaido [44], who investigated the binding of a few dozen compounds to the $\mathrm{DP}_{\mathrm{T}}$ of AcrB. Even if not explicitly mentioned by the authors, a link between the MWs of compounds and their binding preference towards different regions of the pocket is detectable from the analysis of their data. Indeed, we noticed that compounds such as doxorubicin, novobiocin, erythromycin, taurocholate, rifampicin, etc., were predicted to be groove binders, while low-MW compounds such as chloramphenicol, SDS, 1-(1-naphthylmethyl)-piperazine, etc., were predicted to be cave binders. Therefore, the present findings could represent a more general feature related to the mechanism by which AcrB achieves its polyspecificity, namely the ability to accommodate for multiple substrates not only at different pockets located in the different monomers of the transporter, but also at different sub-pockets within the same multi-functional site [42,47].

Unfortunately, we were unable to find any additional parameter correlating with the binding preferences of the carbapenems investigated in this work. However, with the limitations of the adopted computational approaches in mind (such as insufficient sampling of the possible conformations of the molecules within the $\mathrm{DP}_{\mathrm{T}}$, intrinsic limitation of force fields in describing microscopic changes that could happen within the pocket upon recognition and binding, to cite a few), in combination with accurate assays to directly measure efflux of substrates $[53,67,74-76]$, we believe that the computational 
protocol presented in this study represents a valuable source of information to experimentalist aiming at rationalizing microbiological data.

\section{Materials and Methods}

\subsection{Molecular Docking}

Following the protocol adopted in previous investigations [55,59], we first performed an extensive blind docking campaign using AutodockVINA (The Scripps Research Institute, La Jolla, CA, USA) [77] by adopting a rectangular search space of size $125 \times 125 \times 110 \AA^{3}$ enclosing the whole portion of the protein potentially exposed to ligands. Flexibility of both docking partners was considered indirectly, by employing ensembles of conformations. For each compound, representative conformations obtained from an explicitly solvated MD simulation of $1 \mu$ s were used (data available at www.dsf.unica.it/ translocation/db) [78], see also Table S2 for a list of some molecular properties). In all cases we considered only the most populated charge/protonation state at physiological $\mathrm{pH}=7.4$ [78]. For the receptor, conformations including X-ray structures as well as derived from $\mu$ s-long MD simulations were considered [42].

The choice of the initial conformations was limited to one pose within the $\mathrm{DP}_{\mathrm{T}}$ for all molecules. Starting poses were selected according to the estimated binding affinity and the fraction of contacts made by each molecule within the $\mathrm{DP}_{\mathrm{T}}$ (residues lining this region are listed in Table S1). We identified the docking poses within the $\mathrm{DP}_{\mathrm{T}}$ as those in contact ( $3.5 \AA$ cut-off distance) with at least $30 \%$ of the total number of residues lining this site. In addition, the position and orientation of the molecules were also taken into account to evaluate the effect on their behavior inside this region. The number of docking poses identified for each compound according to this criterion, together with the corresponding "average binding affinity" calculated from the scoring function of AutodockVINA [77] are reported in Table S2. Importantly, a statistical contact analysis performed for compounds co-crystallized with AcrB revealed how the adopted docking protocol was able to identify the relevant residues in contact with the ligand in each of the X-ray structures considered (data not shown). The 2D interaction patterns of the selected docking poses for each compound are shown in Figure S4.

\subsection{Simulations and Binding Free-Energy Calculations}

The initial coordinates of the AcrB-molecule complexes were taken from selected docking poses. Due to the large number of compounds considered, we employed a reduced model of the AcrB protein that does not contain the transmembrane domains, and consequently does not require modelling the phospholipidic membrane. This model has been validated in previous studies [36,37]. Each system was immersed in a box containing TIP3P water molecules [79] and an adequate number of $\mathrm{K}^{+}$counterions, in order to neutralize the negative net charge of the system. An osmolarity of $0.15 \mathrm{M}$ was reached by adding an appropriate number of $\mathrm{K}^{+} / \mathrm{Cl}^{-}$. The ff14SB version of the AMBER force field [80] were adopted for AcrB, while the General Amber Force-Field parameters [81] adopted for each molecule were taken from Ref. [78]. The systems were then minimized with a combination of steepest descent and conjugate gradient methods gradually releasing positional restraints applied. The AcrB/molecule complexes were heated from 0 to $310 \mathrm{~K}$ in two steps: a $1 \mathrm{~ns}$ heating from 0 to $100 \mathrm{~K}$ in a canonical ensemble (NVT), followed by $5 \mathrm{~ns}$ heating to reach $310 \mathrm{~K}$ in an isothermal-isobaric ensemble (NPT). Multiple equilibration steps of 500 ps each until the stabilization of the box dimensions were performed in the NPT ensemble. A Langevin thermostat using a collision frequency of $1 \mathrm{ps}^{-1}$ and a Berendsen isotropic barostat [82] maintained a constant temperature, and an average pressure of $1 \mathrm{~atm}$, respectively. A time step of $2 \mathrm{fs}$ was used during the equilibration protocol. The $100 \mathrm{~ns}$ long MD simulations were carried out using the PMEMD module of AMBER 18 (University of California, San Francisco, CA, USA) [83] with a time step of 4 fs in the NVT ensemble, after application of the hydrogen mass repartitioning [84]. Coordinates were saved every 100 ps. Long-range electrostatic interactions were calculated using the particle mesh Ewald method with a cut-off of $9 \AA$ [85] 
Root mean square deviations (RMSDs) were calculated using the cpptraj module of AMBERTools and VMD (Beckman Institute, University of Illinois at Urbana-Champaign, USA) [86]. A cluster analysis using the cpptraj module of AMBERTools identified the most populated configurations sampled during the simulation with a fixed clustering radius of $3.5 \AA$. Only the most populated cluster were further taken into account for evaluating the free energies of binding using the molecular mechanics generalized Born surface area (MM-GBSA [87]) approach. The complementarity of the hydrophobic and hydrophilic properties of the compounds and the $\mathrm{DP}_{\mathrm{T}}$ has been evaluated using PLATINUM webserver [88]. A more detailed description of the MM-GBSA method and the hydrophobic surface analysis implemented in PLATINUM can be found in the Supporting Information of Ref. [55,59]. The starting X-ray crystal configurations and the selected docking poses as well as the "final" cluster representative of each MD simulation can be visualized on-line through the NGL-viewer [89] at www.dsf.unica.it/translocation/docking.

\section{Conclusions}

In this work we combined different computational techniques to investigate the interaction of carbapenem antibiotics with the experimentally known periplasmic binding pocket $\mathrm{DP}_{\mathrm{T}}$ of $\mathrm{AcrB}$, the major efflux transporter from E. coli. Despite the acknowledged limitations, our protocol was able to identify specific residues within the $\mathrm{DP}_{\mathrm{T}}$ of $\mathrm{AcrB}$ playing a prominent role in the binding affinity of selected members of the carbapenem family. Importantly, this study allowed the identification of subtle but relevant differences in compound/AcrB interactions, which can be hardly detected by other means (namely MIC measurements). As such, it proved to be a precious tool to rationalize molecular recognition of carbapenems by AcrB at an atomistic level. Specifically, we highlighted a correlation between the molecular weight of compounds and their binding preference to previously identified sub-pockets within the $\mathrm{DP}_{\mathrm{T}}$, which could represent a general feature of the polyspecificity of AcrB. Altogether, these pieces of information could help in the design of compounds that are less affected by the activity of efflux pumps, or inhibitors able to hinder their function.

Supplementary Materials: Supplementary materials can be found at http://www.mdpi.com/1422-0067/21/3/860/s1.

Author Contributions: Conceptualization, A.A., G.M, A.V.V., and P.R.; methodology, A.A., G.M., F.C., A.B., A.V.V., and P.R..; software, A.A., G.M., A.V.V., and A.B.; validation, A.A., G.M., F.C., and A.V.V.; formal analysis, A.A., G.M., F.C., and A.V.V.; investigation, A.A., G.M., and F.C.; writing original draft, A.A. and G.M.; writing-review and editing, A.A., G.M., F.C., A.B., A.V.V., and P.R.; visualization, A.A., G.M., F.C., and A.V.V.; project administration, P.R.; funding acquisition G.M., A.V.V., and P.R. All authors have read and agreed to the published version of the manuscript.

Funding: The research leading to these results has received support from the National Institutes of Allergy and Infectious Diseases project number AI136799 and the Innovative Medicines Initiatives Joint Undertaking under Grant Agreement number 115525 resources which are composed of financial contribution from the European Union 7th framework programme (FP7/2007-2013) and EFPIA companies in kind contribution. The Authors acknowledge K.M. Pos and J. Dreier for fruitful discussions and G. Serra for technical assistance.

Conflicts of Interest: The authors declare no conflict of interest.

\section{Abbreviations}

AP
DP
EG
HP-Trap
MD
MDR
MIC
MW
RMSD
RND
TM

Access Pocket

Distal Pocket

Exit Gate

Hydrophobic Trap

Molecular Dynamics

Multi Drug Resistance

Minimum Inhibitory Concentration

Molecular Weight

Root Mean Square Displacement

Resistance Nodulation and cell Division

Transmembrane 


\section{References}

1. Paterson, D.L. Impact of antibiotic resistance in gram-negative bacilli on empirical and definitive antibiotic therapy. Clin. Infect. Dis. 2008, 47, S14-S20. [CrossRef]

2. Nikaido, H. Multidrug resistance in bacteria. Annu. Rev. Biochem. 2009, 78, 119-146. [CrossRef]

3. Mehrad, B.; Clark, N.M.; Zhanel, G.G.; Lynch, J.P., 3rd. Antimicrobial resistance in hospital-acquired gram-negative bacterial infections. Chest 2015, 147, 1413-1421. [CrossRef]

4. Piddock, L.J. Clinically relevant chromosomally encoded multidrug resistance efflux pumps in bacteria. Clin. Microbiol. Rev. 2006, 19, 382-402. [CrossRef]

5. Anes, J.; McCusker, M.P.; Fanning, S.; Martins, M. The ins and outs of RND efflux pumps in Escherichia coli. Front. Microbiol. 2015, 6, 587. [CrossRef]

6. Li, X.Z.; Plesiat, P.; Nikaido, H. The challenge of efflux-mediated antibiotic resistance in Gram-negative bacteria. Clin. Microbiol. Rev. 2015, 28, 337-418. [CrossRef] [PubMed]

7. Schindler, B.D.; Kaatz, G.W. Multidrug efflux pumps of Gram-positive bacteria. Drug Resist. Updat. 2016, 27, 1-13. [CrossRef] [PubMed]

8. Du, D.; Wang-Kan, X.; Neuberger, A.; van Veen, H.W.; Pos, K.M.; Piddock, L.J.V.; Luisi, B.F. Multidrug efflux pumps: Structure, function and regulation. Nat. Rev. Microbiol. 2018, 16, 523-539. [CrossRef] [PubMed]

9. Poole, K. Efflux-mediated antimicrobial resistance. J. Antimicrob. Chemother. 2005, 56, 20-51. [CrossRef]

10. Terzi, H.A.; Kulah, C.; Ciftci, I.H. The effects of active efflux pumps on antibiotic resistance in Pseudomonas aeruginosa. World J. Microbiol. Biotechnol. 2014, 30, 2681-2687. [CrossRef]

11. Okusu, H.; Ma, D.; Nikaido, H. AcrAB efflux pump plays a major role in the antibiotic resistance phenotype of Escherichia coli multiple-antibiotic-resistance (Mar) mutants. J. Bacteriol. 1996, 178, 306-308. [CrossRef] [PubMed]

12. Delmar, J.A.; Su, C.C.; Yu, E.W. Bacterial multidrug efflux transporters. Annu. Rev. Biophys. 2014, 43, 93-117. [CrossRef] [PubMed]

13. Seeger, M.A.; Schiefner, A.; Eicher, T.; Verrey, F.; Diederichs, K.; Pos, K.M. Structural asymmetry of AcrB trimer suggests a peristaltic pump mechanism. Science 2006, 313, 1295-1298. [CrossRef] [PubMed]

14. Murakami, S.; Nakashima, R.; Yamashita, E.; Matsumoto, T.; Yamaguchi, A. Crystal structures of a multidrug transporter reveal a functionally rotating mechanism. Nature 2006, 443, 173-179. [CrossRef]

15. Sennhauser, G.; Amstutz, P.; Briand, C.; Storchenegger, O.; Grutter, M.G. Drug export pathway of multidrug exporter AcrB revealed by DARPin inhibitors. PLoS Biol. 2007, 5, e7. [CrossRef]

16. Nakashima, R.; Sakurai, K.; Yamasaki, S.; Nishino, K.; Yamaguchi, A. Structures of the multidrug exporter AcrB reveal a proximal multisite drug-binding pocket. Nature 2011, 480, 565-569. [CrossRef]

17. Ruggerone, P.; Murakami, S.; Pos, K.M.; Vargiu, A.V. RND efflux pumps: Structural information translated into function and inhibition mechanisms. Curr. Top. Med. Chem. 2013, 13, 3079-3100. [CrossRef]

18. Du, D.; Wang, Z.; James, N.R.; Voss, J.E.; Klimont, E.; Ohene-Agyei, T.; Venter, H.; Chiu, W.; Luisi, B.F. Structure of the AcrAB-TolC multidrug efflux pump. Nature 2014, 509, 512-515. [CrossRef]

19. Yamaguchi, A.; Nakashima, R.; Sakurai, K. Structural basis of RND-type multidrug exporters. Front. Microbiol. 2015, 6, 327. [CrossRef]

20. Eicher, T.; Seeger, M.A.; Anselmi, C.; Zhou, W.; Brandstatter, L.; Verrey, F.; Diederichs, K.; Faraldo-Gomez, J.D.; Pos, K.M. Coupling of remote alternating-access transport mechanisms for protons and substrates in the multidrug efflux pump AcrB. Elife 2014, 3, e03145. [CrossRef]

21. Murakami, S.; Nakashima, R.; Yamashita, E.; Yamaguchi, A. Crystal structure of bacterial multidrug efflux transporter AcrB. Nature 2002, 419, 587-593. [CrossRef] [PubMed]

22. Yu, E.W.; McDermott, G.; Zgurskaya, H.I.; Nikaido, H.; Koshland, D.E., Jr. Structural basis of multiple drug-binding capacity of the AcrB multidrug efflux pump. Science 2003, 300, 976-980. [CrossRef] [PubMed]

23. Pos, K.M.; Schiefner, A.; Seeger, M.A.; Diederichs, K. Crystallographic analysis of AcrB. FEBS Lett. 2004, 564, 333-339. [CrossRef]

24. Yu, E.W.; Aires, J.R.; McDermott, G.; Nikaido, H. A periplasmic drug-binding site of the AcrB multidrug efflux pump: A crystallographic and site-directed mutagenesis study. J. Bacteriol. 2005, 187, 6804-6815. [CrossRef] [PubMed] 
25. Seeger, M.A.; von Ballmoos, C.; Eicher, T.; Brandstatter, L.; Verrey, F.; Diederichs, K.; Pos, K.M. Engineered disulfide bonds support the functional rotation mechanism of multidrug efflux pump AcrB. Nat. Struct. Mol. Biol. 2008, 15, 199-205. [CrossRef] [PubMed]

26. Oswald, C.; Tam, H.K.; Pos, K.M. Transport of lipophilic carboxylates is mediated by transmembrane helix 2 in multidrug transporter AcrB. Nat. Commun. 2016, 7, 13819. [CrossRef]

27. Schulz, R.; Vargiu, A.V.; Collu, F.; Kleinekathofer, U.; Ruggerone, P. Functional rotation of the transporter AcrB: Insights into drug extrusion from simulations. PLoS Comput. Biol. 2010, 6, e1000806. [CrossRef]

28. Mishima, H.; Oshima, H.; Yasuda, S.; Kinoshita, M. Statistical thermodynamics for functionally rotating mechanism of the multidrug efflux transporter AcrB. J. Phys. Chem. B 2015, 119, 3423-3433. [CrossRef]

29. Matsunaga, Y.; Yamane, T.; Terada, T.; Moritsugu, K.; Fujisaki, H.; Murakami, S.; Ikeguchi, M.; Kidera, A. Energetics and conformational pathways of functional rotation in the multidrug transporter AcrB. Elife 2018, 7, e31715. [CrossRef]

30. Vargiu, A.V.; Ramaswamy, V.K.; Malvacio, I.; Malloci, G.; Kleinekathofer, U.; Ruggerone, P. Water-mediated interactions enable smooth substrate transport in a bacterial efflux pump. Biochim. Biophys. Acta Gen. Subj. 2018, 1862, 836-845. [CrossRef]

31. Yamane, T.; Murakami, S.; Ikeguchi, M. Functional rotation induced by alternating protonation states in the multidrug transporter AcrB: All-atom molecular dynamics simulations. Biochemistry 2013, 52, 7648-7658. [CrossRef] [PubMed]

32. Eicher, T.; Cha, H.-j.; Seeger, M.A.; Brandstätter, L.; El-Delik, J.; Bohnert, J.A.; Kern, W.V.; Verrey, F.; Grütter, M.G.; Diederichs, K.; et al. Transport of drugs by the multidrug transporter AcrB involves an access and a deep binding pocket that are separated by a switch-loop. Proc. Natl. Acad. Sci. USA 2012, 109, 5687-5692. [CrossRef] [PubMed]

33. Sakurai, K.; Yamasaki, S.; Nakao, K.; Nishino, K.; Yamaguchi, A.; Nakashima, R. Crystal structures of multidrug efflux pump MexB bound with high-molecular-mass compounds. Sci. Rep. 2019, 9, 4359. [CrossRef] [PubMed]

34. Nakashima, R.; Sakurai, K.; Yamasaki, S.; Hayashi, K.; Nagata, C.; Hoshino, K.; Onodera, Y.; Nishino, K.; Yamaguchi, A. Structural basis for the inhibition of bacterial multidrug exporters. Nature 2013, 500, 102-106. [CrossRef]

35. Vargiu, A.V.; Ruggerone, P.; Opperman, T.J.; Nguyen, S.T.; Nikaido, H. Molecular mechanism of MBX2319 inhibition of Escherichia coli AcrB multidrug efflux pump and comparison with other inhibitors. Antimicrob. Agents Chemother. 2014, 58, 6224-6234. [CrossRef]

36. Sjuts, H.; Vargiu, A.V.; Kwasny, S.M.; Nguyen, S.T.; Kim, H.S.; Ding, X.; Ornik, A.R.; Ruggerone, P.; Bowlin, T.L.; Nikaido, H.; et al. Molecular basis for inhibition of AcrB multidrug efflux pump by novel and powerful pyranopyridine derivatives. Proc. Natl. Acad. Sci. USA 2016, 113, 3509-3514. [CrossRef]

37. Vargiu, A.V.; Nikaido, H. Multidrug binding properties of the AcrB efflux pump characterized by molecular dynamics simulations. Proc. Natl. Acad. Sci. USA 2012, 109, 20637-20642. [CrossRef]

38. Kinana, A.D.; Vargiu, A.V.; Nikaido, H. Effect of site-directed mutations in multidrug efflux pump AcrB examined by quantitative efflux assays. Biochem. Biophys. Res. Commun. 2016, 480, 552-557. [CrossRef]

39. Wang, Z.; Fan, G.; Hryc, C.F.; Blaza, J.N.; Serysheva, I.I.; Schmid, M.F.; Chiu, W.; Luisi, B.F.; Du, D. An allosteric transport mechanism for the AcrAB-TolC multidrug efflux pump. Elife 2017, 6. [CrossRef]

40. Ababou, A. New insights into the structural and functional involvement of the gate loop in AcrB export activity. Biochim. Biophys. Acta 2018, 1866, 242-253. [CrossRef]

41. Fischer, N.; Kandt, C. Porter domain opening and closing motions in the multi-drug efflux transporter AcrB. Biochim. Biophys. Acta 2013, 1828, 632-641. [CrossRef] [PubMed]

42. Ramaswamy, V.K.; Vargiu, A.V.; Malloci, G.; Dreier, J.; Ruggerone, P. Molecular Rationale behind the Differential Substrate Specificity of Bacterial RND Multi-Drug Transporters. Sci. Rep. 2017, 7, 8075. [CrossRef] [PubMed]

43. Li, J.; Wen, P.C.; Moradi, M.; Tajkhorshid, E. Computational characterization of structural dynamics underlying function in active membrane transporters. Curr. Opin. Struct. Biol. 2015, 31, 96-105. [CrossRef] [PubMed]

44. Takatsuka, Y.; Chen, C.; Nikaido, H. Mechanism of recognition of compounds of diverse structures by the multidrug efflux pump AcrB of Escherichia coli. Proc. Natl. Acad. Sci. USA 2010, 107, 6559-6565. [CrossRef] [PubMed] 
45. Kobylka, J.; Kuth, M.S.; Muller, R.T.; Geertsma, E.R.; Pos, K.M. AcrB: A mean, keen, drug efflux machine. Ann. N Y Acad. Sci. 2020, 1459, 38-68. [CrossRef]

46. Yao, X.Q.; Kimura, N.; Murakami, S.; Takada, S. Drug uptake pathways of multidrug transporter AcrB studied by molecular simulations and site-directed mutagenesis experiments. J. Am. Chem. Soc. 2013, 135, 7474-7485. [CrossRef]

47. Imai, T.; Miyashita, N.; Sugita, Y.; Kovalenko, A.; Hirata, F.; Kidera, A. Functionality mapping on internal surfaces of multidrug transporter AcrB based on molecular theory of solvation: Implications for drug efflux pathway. J. Phys. Chem. B 2011, 115, 8288-8295. [CrossRef]

48. Nicolau, D.P. Carbapenems: A potent class of antibiotics. Expert. Opin. Pharmacother. 2008, 9, $23-37$. [CrossRef]

49. Papp-Wallace, K.M.; Endimiani, A.; Taracila, M.A.; Bonomo, R.A. Carbapenems: Past, present, and future. Antimicrob. Agents Chemother. 2011, 55, 4943-4960. [CrossRef]

50. Okamoto, K.; Gotoh, N.; Nishino, T. Alterations of susceptibility of Pseudomonas aeruginosa by overproduction of multidrug efflux systems, MexAB-OprM, MexCD-OprJ, and MexXY/OprM to carbapenems: Substrate specificities of the efflux systems. J. Infect. Chemother. 2002, 8, 371-373. [CrossRef]

51. Li, X.Z.; Ma, D.; Livermore, D.M.; Nikaido, H. Role of efflux pump(s) in intrinsic resistance of Pseudomonas aeruginosa: Active efflux as a contributing factor to beta-lactam resistance. Antimicrob. Agents Chemother. 1994, 38, 1742-1752. [CrossRef] [PubMed]

52. Kohler, T.; Michea-Hamzehpour, M.; Epp, S.F.; Pechere, J.C. Carbapenem activities against Pseudomonas aeruginosa: Respective contributions of OprD and efflux systems. Antimicrob. Agents Chemother. 1999, 43, 424-427. [CrossRef] [PubMed]

53. Blair, J.M.; Piddock, L.J. How to Measure Export via Bacterial Multidrug Resistance Efflux Pumps. mBio 2016, 7, e00840-00816. [CrossRef] [PubMed]

54. Krishnamoorthy, G.; Wolloscheck, D.; Weeks, J.W.; Croft, C.; Rybenkov, V.V.; Zgurskaya, H.I. Breaking the Permeability Barrier of Escherichia coli by Controlled Hyperporination of the Outer Membrane. Antimicrob. Agents Chemother. 2016, 60, 7372-7381. [CrossRef] [PubMed]

55. Atzori, A.; Malviya, V.N.; Malloci, G.; Dreier, J.; Pos, K.M.; Vargiu, A.V.; Ruggerone, P. Identification and characterization of carbapenem binding sites within the RND-transporter AcrB. Biochim. Biophys. Acta Biomembr. 2019, 1861, 62-74. [CrossRef]

56. Iyer, R.; Erwin, A.L. Direct measurement of efflux in Pseudomonas aeruginosa using an environment-sensitive fluorescent dye. Res. Microbiol. 2015, 166, 516-524. [CrossRef]

57. Cinquin, B.; Maigre, L.; Pinet, E.; Chevalier, J.; Stavenger, R.A.; Mills, S.; Réfrégiers, M.; Pagès, J.-M. Microspectrometric insights on the uptake of antibiotics at the single bacterial cell level. Sci. Rep. 2015, 5, 17968. [CrossRef]

58. Nagano, K.; Nikaido, H. Kinetic behavior of the major multidrug efflux pump AcrB of Escherichia coli. Proc. Natl. Acad. Sci. USA 2009, 106, 5854-5858. [CrossRef]

59. Atzori, A.; Malloci, G.; Prajapati, J.D.; Basciu, A.; Bosin, A.; Kleinekathofer, U.; Dreier, J.; Vargiu, A.V.; Ruggerone, P. Molecular Interactions of Cephalosporins with the Deep Binding Pocket of the RND Transporter AcrB. J. Phys. Chem. B 2019, 123, 4625-4635. [CrossRef]

60. Malvacio, I.; Buonfiglio, R.; D'Atanasio, N.; Serra, G.; Bosin, A.; Di Giorgio, F.P.; Ruggerone, P.; Ombrato, R.; Vargiu, A.V. Molecular basis for the different interactions of congeneric substrates with the polyspecific transporter AcrB. Biochim. Biophys. Acta Biomembr. 2019, 1861, 1397-1408. [CrossRef]

61. Collu, F.; Vargiu, A.V.; Dreier, J.; Cascella, M.; Ruggerone, P. Recognition of imipenem and meropenem by the RND-transporter MexB studied by computer simulations. J. Am. Chem. Soc. 2012, 134, 19146-19158. [CrossRef] [PubMed]

62. Husain, F.; Nikaido, H. Substrate path in the AcrB multidrug efflux pump of Escherichia coli. Mol. Microbiol. 2010, 78, 320-330. [CrossRef] [PubMed]

63. Lim, S.P.; Nikaido, H. Kinetic parameters of efflux of penicillins by the multidrug efflux transporter AcrAB-TolC of Escherichia coli. Antimicrob. Agents Chemother. 2010, 54, 1800-1806. [CrossRef] [PubMed]

64. Kinana, A.D.; Vargiu, A.V.; May, T.; Nikaido, H. Aminoacyl beta-naphthylamides as substrates and modulators of AcrB multidrug efflux pump. Proc. Natl. Acad. Sci. USA 2016, 113, 1405-1410. [CrossRef] 
65. Adler, M.; Anjum, M.; Andersson, D.I.; Sandegren, L. Combinations of mutations in envZ, ftsI, mrdA, acrB and acrR can cause high-level carbapenem resistance in Escherichia coli. J. Antimicrob. Chemother. 2016, 71, 1188-1198. [CrossRef]

66. Bohnert, J.A.; Schuster, S.; Seeger, M.A.; Fahnrich, E.; Pos, K.M.; Kern, W.V. Site-directed mutagenesis reveals putative substrate binding residues in the Escherichia coli RND efflux pump AcrB. J. Bacteriol. 2008, 190, 8225-8229. [CrossRef]

67. Bohnert, J.A.; Schuster, S.; Szymaniak-Vits, M.; Kern, W.V. Determination of real-time efflux phenotypes in Escherichia coli AcrB binding pocket phenylalanine mutants using a 1,2'-dinaphthylamine efflux assay. PLoS One 2011, 6, e21196. [CrossRef]

68. Nishino, K.; Yamada, J.; Hirakawa, H.; Hirata, T.; Yamaguchi, A. Roles of TolC-dependent multidrug transporters of Escherichia coli in resistance to beta-lactams. Antimicrob. Agents Chemother. 2003, 47, 3030-3033. [CrossRef]

69. Saw, H.T.; Webber, M.A.; Mushtaq, S.; Woodford, N.; Piddock, L.J. Inactivation or inhibition of AcrAB-TolC increases resistance of carbapenemase-producing Enterobacteriaceae to carbapenems. J. Antimicrob. Chemother. 2016, 71, 1510-1519. [CrossRef]

70. Cooper, S.J.; Krishnamoorthy, G.; Wolloscheck, D.; Walker, J.K.; Rybenkov, V.V.; Parks, J.M.; Zgurskaya, H.I. Molecular Properties That Define the Activities of Antibiotics in Escherichia coli and Pseudomonas aeruginosa. ACS Infect. Dis. 2018, 4, 1223-1234. [CrossRef]

71. Ramaswamy, V.K.; Vargiu, A.V.; Malloci, G.; Dreier, J.; Ruggerone, P. Molecular Determinants of the Promiscuity of MexB and MexY Multidrug Transporters of Pseudomonas aeruginosa. Front. Microbiol. 2018, 9, 1144. [CrossRef] [PubMed]

72. Jamshidi, S.; Sutton, J.M.; Rahman, K.M. Computational Study Reveals the Molecular Mechanism of the Interaction between the Efflux Inhibitor PAbetaN and the AdeB Transporter from Acinetobacter baumannii. ACS Omega 2017, 2, 3002-3016. [CrossRef] [PubMed]

73. Vargiu, A.V.; Ramaswamy, V.K.; Malloci, G.; Malvacio, I.; Atzori, A.; Ruggerone, P. Computer simulations of the activity of RND efflux pumps. Res. Microbiol. 2018, 169, 384-392. [CrossRef] [PubMed]

74. Whittle, E.E.; Legood, S.W.; Alav, I.; Dulyayangkul, P.; Overton, T.W.; Blair, J.M.A. Flow Cytometric Analysis of Efflux by Dye Accumulation. Front. Microbiol. 2019, 10, 2319. [CrossRef]

75. Bohnert, J.A.; Karamian, B.; Nikaido, H. Optimized Nile Red efflux assay of AcrAB-TolC multidrug efflux system shows competition between substrates. Antimicrob. Agents Chemother. 2010, 54, 3770-3775. [CrossRef] [PubMed]

76. Vergalli, J.; Dumont, E.; Cinquin, B.; Maigre, L.; Pajovic, J.; Bacque, E.; Mourez, M.; Refregiers, M.; Pages, J.M. Fluoroquinolone structure and translocation flux across bacterial membrane. Sci. Rep. 2017, 7, 9821. [CrossRef] [PubMed]

77. Trott, O.; Olson, A.J. AutoDock Vina: Improving the speed and accuracy of docking with a new scoring function, efficient optimization, and multithreading. J. Comput. Chem. 2010, 31, 455-461. [CrossRef]

78. Malloci, G.; Vargiu, A.V.; Serra, G.; Bosin, A.; Ruggerone, P.; Ceccarelli, M. A Database of Force-Field Parameters, Dynamics, and Properties of Antimicrobial Compounds. Molecules 2015, 20, 13997-14021. [CrossRef]

79. Jorgensen, W.L.; Chandrasekhar, J.; Madura, J.D.; Impey, R.W.; Klein, M.L. Comparison of simple potential functions for simulating liquid water. J. Chem. Phys. 1983, 79, 926-935. [CrossRef]

80. Maier, J.A.; Martinez, C.; Kasavajhala, K.; Wickstrom, L.; Hauser, K.E.; Simmerling, C. ff14SB: Improving the Accuracy of Protein Side Chain and Backbone Parameters from ff99SB. J. Chem. Theory. Comput. 2015, 11, 3696-3713. [CrossRef]

81. Wang, J.; Wolf, R.M.; Caldwell, J.W.; Kollman, P.A.; Case, D.A. Development and testing of a general amber force field. J. Comput. Chem. 2004, 25, 1157-1174. [CrossRef] [PubMed]

82. Berendsen, H.J.C.; Postma, J.P.M.; van Gunsteren, W.F.; DiNola, A.; Haak, J.R. Molecular dynamics with coupling to an external bath. J. Chem. Phys. 1984, 81, 3684-3690. [CrossRef]

83. AMBER 18; Case, D.A.; Ben-Shalom, I.Y.; Brozell, S.R.; Cerutti, D.S.; Cheatham, T.E., III; Cruzeiro, V.W.D.; Darden, T.A.; Duke, R.E.; Ghoreishi, D.; Gilson, M.K.; et al. (Eds.) University of California: San Francisco, CA, USA, 2018.

84. Feenstra, K.A.; Hess, B.; Berendsen, H.J.C. Improving efficiency of large time-scale molecular dynamics simulations of hydrogen-rich systems. J. Comput. Chem. 1999, 20, 786-798. [CrossRef] 
85. Darden, T.; York, D.; Pedersen, L. Particle mesh Ewald: An N·log(N) method for Ewald sums in large systems. J. Chem. Phys. 1993, 98, 10089-10092. [CrossRef]

86. Humphrey, W.; Dalke, A.; Schulten, K. VMD: Visual molecular dynamics. J. Mol. Graph. 1996, 14, 33-38. [CrossRef]

87. Kollman, P.A.; Massova, I.; Reyes, C.; Kuhn, B.; Huo, S.; Chong, L.; Lee, M.; Lee, T.; Duan, Y.; Wang, W.; et al. Calculating structures and free energies of complex molecules: Combining molecular mechanics and continuum models. Acc. Chem. Res. 2000, 33, 889-897. [CrossRef]

88. Pyrkov, T.V.; Chugunov, A.O.; Krylov, N.A.; Nolde, D.E.; Efremov, R.G. PLATINUM: A web tool for analysis of hydrophobic/hydrophilic organization of biomolecular complexes. Bioinformatics 2009, 25, 1201-1202. [CrossRef]

89. Rose, A.S.; Bradley, A.R.; Valasatava, Y.; Duarte, J.M.; Prlic, A.; Rose, P.W. NGL viewer: Web-based molecular graphics for large complexes. Bioinformatics 2018, 34, 3755-3758. [CrossRef]

(C) 2020 by the authors. Licensee MDPI, Basel, Switzerland. This article is an open access article distributed under the terms and conditions of the Creative Commons Attribution (CC BY) license (http://creativecommons.org/licenses/by/4.0/). 